\title{
Research on Aerodynamic Modeling of Elman Neural Network Based on PSO Algorithm
}

\author{
Gan Xusheng ${ }^{1, a}$, Wang Minghua ${ }^{2, b}$, Li Huaping ${ }^{3, c}$ \\ ${ }^{1 .}$ XiJing College, Xi'an, Shaanxi, 710123, China; \\ 2. Air Traffic Control and Navigation College, Air Force Engineering University, Xi'an, Shaanxi, 710051, \\ China; \\ 3. PLA, Air Force, Xi' an Flight Academy, Xi'an, Shaanxi, 710306 \\ aganxusheng123@163.com; bwmh516@163.com; ㄷw2121@sohu.com
}

Keywords: Elman Neural Network, Particle Swarm Optimization, Aircraft, Aerodynamic Modeling.

\begin{abstract}
For the accurate description of aerodynamic characteristics for aircraft, an Elman Neural Network (ENN) aerodynamic modeling method, based on Particle Swarm Optimization (PSO) algorithm, is proposed. In the method, PSO algorithm is introduced to optimize the parameters of ENN. Simulation results indicate that the aerodynamic modeling method proposed can achieve the accuracy improvement compared with GD-NN, RBF-NN and GD-ENN, and can converge to a satisfactory precision by $60 \sim 120$ iterations, it has been proved that the proposed method is feasible and effective for aerodynamic modeling from flight data.
\end{abstract}

\section{Introduction}

The accuracy of the aerodynamic model is the foundation of the performance analysis and hardware -in-loop Simulation of the aircraft. With flight simulator as an example, whether the aerodynamic model built can accurately describe the dynamic characteristic of aircraft is the important prerequisite of the simulation with great reality. Considering that the wind tunnel experiments cannot really simulate the actual flight state for the aircraft with great expense, we focus on using the modern control theory and identification technology to establish the aerodynamic model from flight test data for aircraft. It is a promising research direction as well as a great economic significance.

Based on this, Elman Neural Network (ENN) trained by PSO algorithm is introduced to establish the aerodynamic model of the aircraft for the accuracy improvement. It also is verified by an example.

\section{Particle Swarm Optimization Algorithm}

PSO algorithm is proposed by Kennedy and Eberhart in 1995, and had been widely used in many fields because of simple operation and high efficiency [1]. Its basic idea is originated from a simplified simulation model of the swarm behavior. On account of rapid information exchange between the particles, PSO algorithm has fast convergence speed.

In the PSO algorithm, each individual is named as a particle which represents a potential solution in the search space. The position vector and velocity vector of $i$-th particle in $N$-dimensional space are represented as $X_{i}=\left(x_{i 1}, x_{i 2}, \cdots, x_{i N}\right), V_{i}=\left(v_{i 1}, v_{i 2}, \cdots, v_{i N}\right)$ respectively. In the search process, each particle knows its best previous position $p_{\text {besti }}$ (with the best fitness value) and current position $X_{i}$, which can be seen as its own flying experience. In addition, each particle also knows the best previous position $g_{\text {best }}$ among all particles in the swarm ( $g_{\text {best }}$ is the optimal value among all $\left.p_{\text {besti }}\right)$, which can be seen as the experiences of neighbors. 
For PSO algorithm, the velocity and position update formulas are as follows

$$
\begin{gathered}
v_{i d}(t+1)=w \cdot v_{i d}(t)+c_{1} \cdot r_{1} \cdot\left(p_{\text {bestid }}(t)-x_{i d}(t)\right)+c_{2} \cdot r_{2} \cdot\left(g_{\text {bestd }}(t)-x_{i d}(t)\right) \\
x_{i d}(t+1)=x_{i d}(t)+v_{i d}(t+1)
\end{gathered}
$$

where $c_{1}, c_{2}$ are acceleration coefficients which are nonnegative constants. $r_{1}, r_{2}$ are generated randomly in $[0,1] . t$ is the current iteration time. $p_{\text {bestid }}$ is the $d$-th dimension of the best position $p_{\text {besti }}$ of the $i$-th particle. $g_{\text {bestd }}$ is the $d$-th dimension of the best particle position $g_{\text {best }}$ among all the particles. $i=1,2, \ldots, M$, and $M$ is the swarm size. $d=1,2, \ldots, N . w$ is the inertia weight.

\section{Modified Elman Neural Network}

ENN proposed by Elman is a typical recurrent neural network [2][3]. A context layer is added in the hidden layer of feed-forward neural network as one step delay operator to achieve the purpose of memory, so that the system has the adaptive ability for time-varying characteristics. Hence it is a kind of feedback dynamics system, and can directly reflect the characteristics of the dynamic process of the system with the calculation ability, which is very suitable for establishing the prediction model of the time series for complicated nonlinear dynamics system.

The structure of ENN is generally composed of input layer, hidden layer, output layer and context layer, as shown in Fig. 1. The connection between input layer, hidden layer and output layer is similar to that of feed-forward neural network. The unit of input layer only plays the role of signal transmission. The unit of output layer plays the role of linear weighting. The transfer function of unit of hidden layer can be taken linear or nonlinear functions. The context layer can be used to memory the output value of unit of hidden layer at last time, which can be considered as one step delay operator.

The main characteristic of ENN is that, the output of hidden layer can connect with the input to hidden layer by delay and storage of context layer, which makes ENN very sensitive to the data of historical state, and the addition of internal feedback network also improve the processing capacity of dynamic information of the network to achieve the purpose of dynamic modeling.



Fig.1. Structure of modified ENN

In modified ENN, a self-feedback connection with a fixed gain value $\delta$ is added on the context layer unit of basic ENN, which the output of the of context layer unit at time $t$ is equal to $\delta$ multiple 
of the sum of the output of hidden layer unit and the output of the of context layer unit at time $t-1$. Compared with basic ENN, the modified ENN can better adjust the strength of feedback signal. The structure of modified ENN is shown in Fig. 1. Among them, $M^{-1}$ is the one step delay operator; $0 \leq$ $\delta<0$ is the self feedback gain factor, when $\delta=0$, it is the basic ENN; when $\delta \neq 0$, it is the modified ENN.

In structure of modified ENN, $w_{a}$ is the connection weight between the input layer and the hidden layer, $w_{b}$ is the connection weight between the context layer and the output layer, $w_{c}$ is the connection weight between the hidden layer and the output layer. $F(\cdot)$ is the transfer function of hidden layer, using the Sigmoid function $F(x)=1 /\left(1+\mathrm{e}^{-x}\right)$ is often used. $\mathrm{G}(\cdot)$ is the linear transfer function of output unit and is a linear combination of output of hidden layer.

The feed-forward connection part of ENN can be mainly conducted to correct the connection weight. The recursive part is fixed, which cannot be corrected. Suppose that the input layer has $r$ nodes, and there are $n$ nodes in the hidden layer and the context layer respectively, in addition, there is one node in the output layer, then the input $u$ of ENN is $r$-dimension vector, the output $x(t)$ of hidden layer and output $x_{c}(t)$ of context layer is $n$-dimension vector, $\delta$ is $n$-dimension vector, the output $y$ of ENN is 1-dimension vector, the connection weight $w_{a}$ is $n \mathrm{X} r$-dimension matrix, $w_{b}$ is $n \mathrm{X} n$-dimension matrix, $w_{c}$ is $1 \mathrm{X} n$-dimension matrix. The mathematical model of ENN can be expressed as

$$
\begin{gathered}
x(t)=F\left(w_{a} u(t-1)\right)+w_{b} x_{c}(t) \\
x_{c}(t)=x(t-1)+\delta \cdot x_{c}(t-1) \\
y(t)=G\left(w_{c} x(t)\right)
\end{gathered}
$$

ENN is essentially, through the feedback signal, storage the inner state to obtain the mapping function of dynamic characteristics based on feed-forward neural network, so that the system has the ability to adapt to the time-varying characteristics, thus the prediction capability of ENN is better than BP neural network for time series. However, for the modification of network weight, ENN still uses the gradient descent algorithm with the deficiency of slow learning speed, easy to be trapped in local minima. Generally, the self-feedback gain factor $\delta$ is obtained by trial method, which leads to the low learning efficiency.

\section{ENN Based on PSO Algorithm}

PSO algorithm does not need to be differentiable on the function. Training the parameters of ENN by it can improve the performance and training speed of the network. In the paper, PSO algorithm is used to train ENN, namely optimize the parameter vector $\theta=\left\{w_{a}, w_{b}, w_{c}, \delta\right\}$ of ENN. Its simplified training process is shown in Fig. 2, where the termination condition is the maximum number of iterations or target error.

\section{Experiment Simulation}

To validate the proposed aerodynamic modeling method, the air flight simulator ATTAS is selected as the object of study, and all measured flight data is from the literature [4].

The experiments of aircraft aerodynamic modeling are divided into lateral modeling and longitudinal modeling. The flight measured data are derived from two maneuvers of the aerial flight simulator ATTAS while the aileron, rudder and elevator activation is input. In experiment, the hidden layer nodes can be taken as 10 . In order to eliminate the influence of dimension, the flight data need to be standardized before modeling. 




Fig. 2. Training flow of PSO-ENN

The lateral aerodynamic model of ATTAS can be expressed as

$$
\left[C_{Y}, C_{l}, C_{n}\right]=f_{\text {lat }}\left(\beta, p, r, \delta_{a}, \delta_{r}\right)
$$

where the outputs are the side force coefficient $C_{\mathrm{Y}}$, the rolling moment coefficient $C_{l}$ and the yaw moment coefficient $C_{n}$ of the aircraft. The inputs are the sideslip angle $\beta$, the roll angular rate $p$, the yaw angular rate $r$, the aileron angle $\delta_{a}$ and the rudder angle $\delta_{r}$ of the aircraft. The relevant variables are shown in Fig. 3.

The longitudinal aerodynamic model of ATTAS can be expressed as

$$
\left[C_{D}, C_{L}, C_{m}\right]=f_{\text {lon }}\left(\alpha, q, V, \delta_{e}\right)
$$

where the outputs are the drag force coefficient $C_{D}$, the lift force coefficient $C_{L}$ and the yaw pitching moment coefficient $C_{m}$ of the aircraft. The inputs are the angle of attack $\alpha$, the pitch angular rate $q$, the true velocity $V$, the elevator angle $\delta_{e}$ of the aircraft.

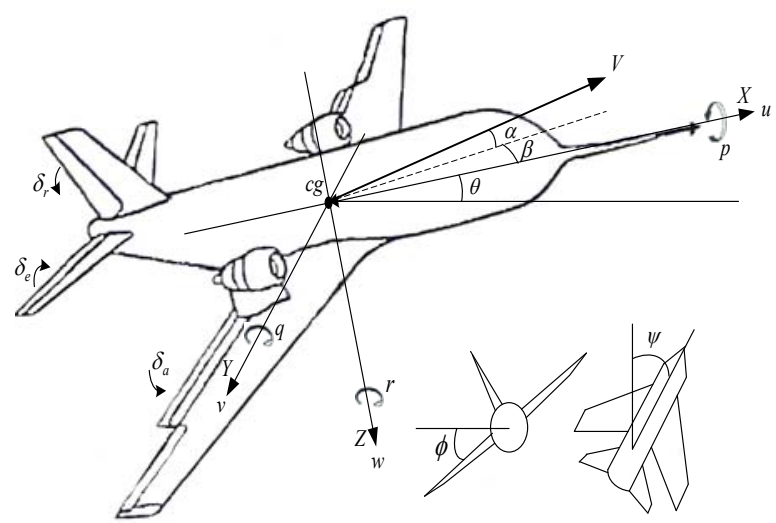

Fig. 3. Graphics explanation on relevant variable symbols

To realize aerodynamic modeling from flight data measured, the aerodynamic force coefficient $C_{\mathrm{Y}}, C_{D}, C_{L}$ and aerodynamic moment coefficient $C_{m}, C_{n}, C_{l}$ usually cannot be measured directly, but can be calculated by the process that is introduced in reference [5].

From the experimental results in Table 1 and Fig. 4 8, it can be concluded that, in test MSE, ENN based on PSO learning algorithm is obviously better than GD-NN and RBF-NN, and is roughly equivalent to GD-ENN, but in training Time (T, unit: second s) PSO-ENN has superiority 
to GD-ENN. This once again validates that the PSO algorithm is used to optimize the parameters of ENN, which can not only maintains good performance using PSO algorithm to train ENN model, but also reduce the computation and learning time. This provides the method support for ENN aerodynamic modeling of the aircraft.

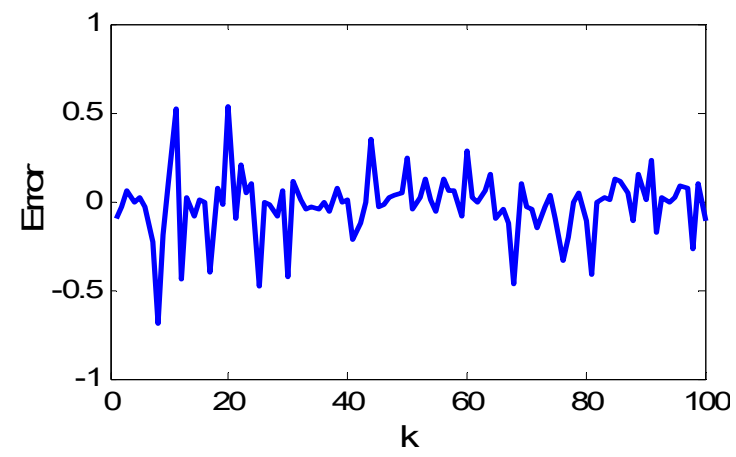

Fig. 4. Prediction error of lateral aerodynamic model

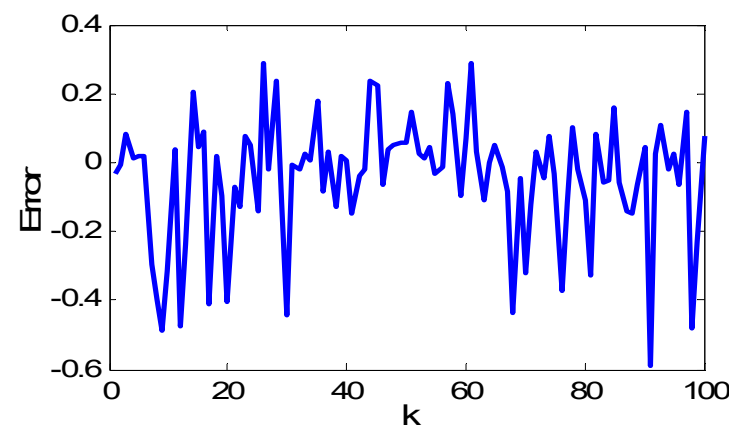

Fig. 5. Prediction error of longitudinal aerodynamic model

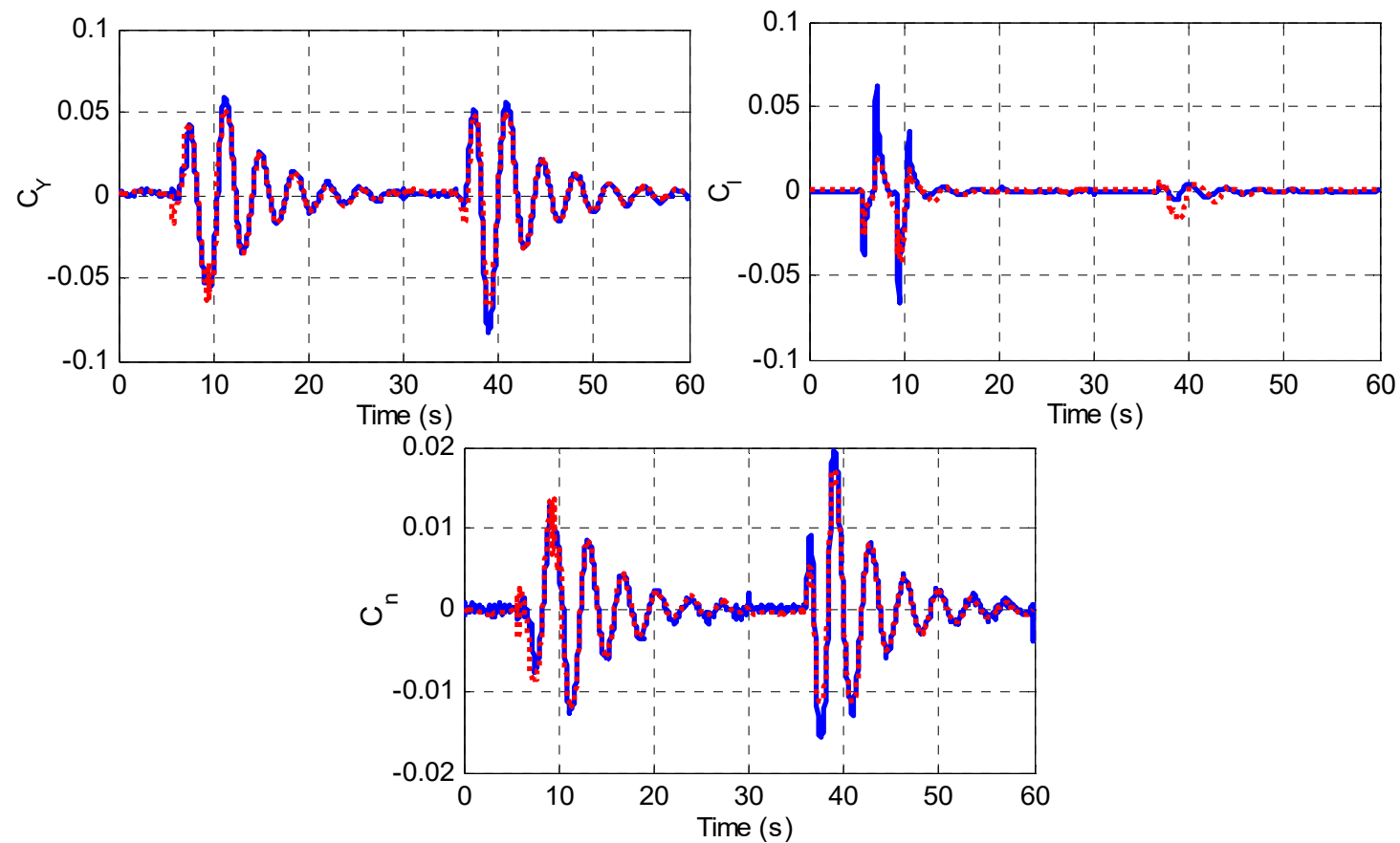

Fig. 6. Prediction results of lateral aerodynamic model

(- flight derived, --- model estimated) 

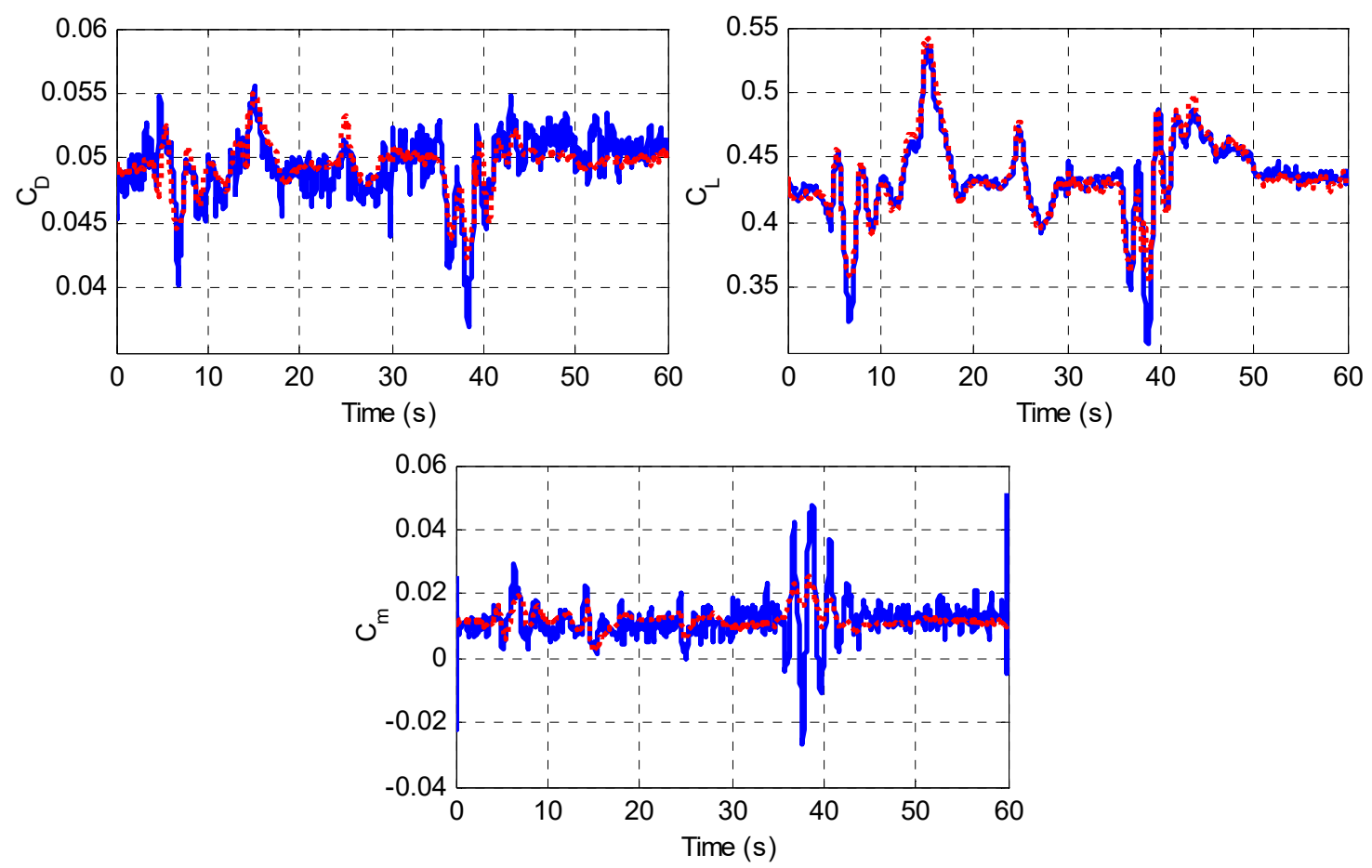

Fig. 7. Prediction results of longitudinal aerodynamic model

(- flight derived, --- model estimated)

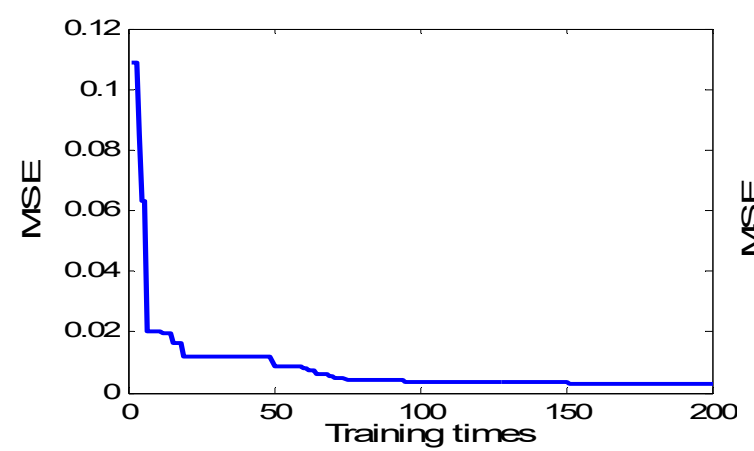

Lateral aerodynamic modeling

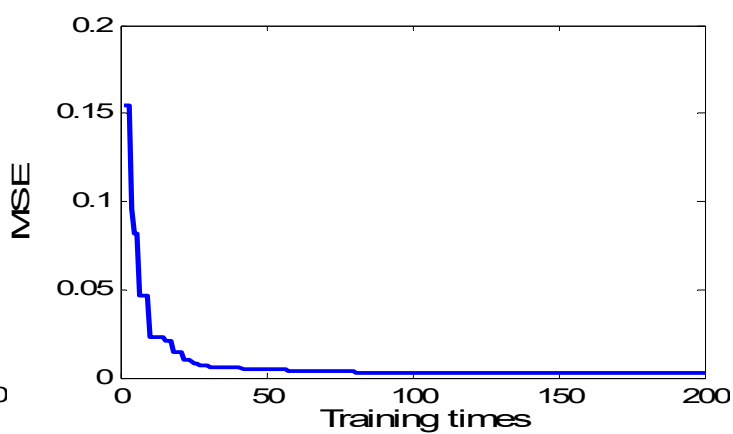

Longitudinal aerodynamic modeling

Fig. 8. Convergence curves of WNN training

Table 1 Comparison of Simulation Results for Aerodynamic Modeling

\begin{tabular}{|c|c|c|c|c|c|c|c|c|c|}
\hline \multirow{2}{*}{\multicolumn{2}{|c|}{$\begin{array}{l}\text { Aerodynamic } \\
\text { modeling }\end{array}$}} & \multicolumn{2}{|c|}{ GD-NN } & \multicolumn{2}{|c|}{ RBF-NN } & \multicolumn{2}{|c|}{ GD-ENN } & \multicolumn{2}{|c|}{ PSO-ENN } \\
\hline & & $\begin{array}{l}\text { Prediction } \\
\text { MSE }\end{array}$ & $\mathrm{T}$ & $\begin{array}{l}\text { Prediction } \\
\text { MSE }\end{array}$ & $\mathrm{T}$ & $\begin{array}{l}\text { Prediction } \\
\text { MSE }\end{array}$ & $\mathrm{T}^{\mathrm{I}}$ & $\begin{array}{l}\text { Prediction } \\
\text { MSE }\end{array}$ & $\mathrm{T}$ \\
\hline \multirow{3}{*}{ 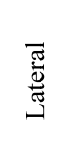 } & $C_{Y}$ & 0.0056 & & 0.0045 & & 0.0026 & & 0.0031 & \\
\hline & $C_{l}$ & 0.0127 & $44 \mathrm{~s}$ & 0.0084 & $71 \mathrm{~s}$ & 0.0045 & $127 \mathrm{~s}$ & 0.0052 & $85 \mathrm{~s}$ \\
\hline & $C_{n}$ & 0.0032 & & 0.0035 & & 0.0013 & & 0.0016 & \\
\hline \multirow{3}{*}{ 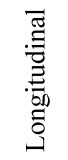 } & $C_{D}$ & 0.0042 & & 0.0046 & & 0.0016 & & 0.0020 & \\
\hline & $C_{L}$ & 0.0175 & $53 \mathrm{~s}$ & 0.0114 & $84 \mathrm{~s}$ & 0.0078 & $143 \mathrm{~s}$ & 0.0087 & $97 \mathrm{~s}$ \\
\hline & $C_{m}$ & 0.0112 & & 0.0091 & & 0.0059 & & 0.0057 & \\
\hline
\end{tabular}

\section{Conclusion}

For the aerodynamic modeling of the aircraft, ENN modeling method based on PSO learning algorithm is proposed. The simulation results show that, the aerodynamic modeling based on PSO-ENN from flight data has the advantages such as precision, convergence and surmounting 
precocity, and can accurately characterize the aerodynamic action of aircraft with easy implementation. Thus PSO-ENN is a very promising method in aerodynamic modeling for aircraft, worthy of in-depth research and concern. At the same time, it also provides a powerful method for modeling and predicting problems of nonlinear systems.

\section{References}

[1] J. Kennedy, R. C. Eberhart, "Particle swarm optimization," Proceedings of 4th IEEE International Conference on Neural Networks, 1995, pp. 1942-1948.

[2] C. Zhang, T. H. Chang, H. Liu, et al, "Wind speed forecast based on improved Elman neural network," East China Electric Power, vol. 40, No. 8, 2012, pp. 1412-1414.

[3] J. L. Elman, "Find structure in time," Cognitive Science, vol. 14, No. 2, 1990, pp. 179-211.

[4] R. V. Jategaonkar, "Flight vehicle system identification: a time domain methodology,"Progress in Astronautics and Aeronautics Series, Published by AIAA, 2006.

[5] X. S. Gan, J. S. Duanmu, W Cong, "Aerodynamic modeling from flight data based on WNN optimized by particle swarm," Acta Aeronautica et Astronautica Sinica, vol. 33, No. 7, 2012, pp. 1209-1929. 